\title{
Gas-rich LSB Galaxies - Progenitors of Blue Compact Dwarfs?
}

\author{
John J. Salzer \\ Astronomy Department, Wesleyan University, Middletown, CT 06459 \\ Stuart A. Norton \\ Lick Observatory, Univ. California Santa Cruz, Santa Cruz, CA 95064
}

\begin{abstract}
We analyze deep CCD images of nearby Blue Compact Dwarf (BCD) galaxies in an attempt to understand the nature of the progenitors which are hosting the current burst of star formation. In particular, we ask whether BCDs are hosted by normal or low-surface-brightness dI galaxies. We conclude that BCDs are in fact hosted by gas-rich galaxies which populate the extreme high-central-mass-density end of the dwarf galaxy distribution. Such galaxies are predisposed to having numerous strong bursts of star formation in their central regions. In this picture, $\mathrm{BCDs}$ can only occur in the minority of dwarf galaxies, rather than being a common phase experienced by all gas-rich dwarfs.
\end{abstract}

\section{Introduction}

If one wished to adopt the simplest scheme for classifying dwarf galaxies, most could be lumped into one of two categories. The first would be the dwarf irregulars (dIs), which can be characterized as having plenty of gas and usually some level of recent or current star formation. The other would be dwarf ellipticals (dEs), which by comparison to dIs have little if any gas, and usually no significant recent star formation (although exceptions to this latter point certainly exist). Interestingly, both types of dwarfs tend to have surface brightness distributions that are well fit by simple exponential profiles. Structural parameters derived from surface photometry of both dIs and dEs show a large range of values: galaxies of both types are observed with both high and low central surface brightnesses $\left(\mu_{0}\right)$, and both large and small exponential scale-lengths $(\alpha)$. In fact, the two types overlap completely in the the $\mu_{0}-\alpha$ plane.

A group of low-luminosity galaxies which do not readily lend themselves to classification in the above scheme are the blue compact dwarfs (BCDs). These are dwarf galaxies which are currently undergoing an extremely strong burst of star formation, such that the optical appearance of the galaxy is dominated by the energy output of the young stars. In some cases the starburst is so dominant that the presence of an underlying older population of stars is not clearly evident.

Given their extreme nature, it has been difficult to determine with any confidence the type of galaxy that typically hosts BCDs. Since BCDs are usually observed to be gas rich (Thaun \& Martin 1983, Salzer et al. 1999a), the most 
common assumption is that BCDs represent bursts of star formation occurring in dI galaxies. But can any $\mathrm{dI}$ initiate a large star-formation episode and appear as a BCD? This is a key question, since it impacts our picture of how dwarf galaxies evolve. Is the $\mathrm{BCD}$ phenomenon a stage of galaxy evolution common to all gas-rich dwarfs? We attempt to answer this question in the current study.

\section{Relevant Facts about BCDs}

Before attempting to address the question posed in the previous section, we review some of the relevant characteristics of BCDs:

- Optical appearance dominated by light from starburst. As mentioned above, BCDs are dwarf galaxies whose optical light output is dominated by the energy released by the starburst component. This includes the light from the young $\mathrm{O}$ and $\mathrm{B}$ stars, plus the nebular emission (both line and continuum) which represents reprocessed UV radiation from the same massive stars. The latter can be a major contributor to the broad-band fluxes measured in BCDs, in extreme cases exceeding the light of the stellar component in the optical. This characteristic of BCDs has made classification of the underlying host galaxy all but impossible in many cases.

- Very intense nebular spectra. Spectra of BCDs are dominated by nebular emission lines. In most cases, strong recombination lines of $\mathrm{H}$ and $\mathrm{He}$ obliterate any stellar absorption lines which may be present. In addition, the nebular continuum combined with the relatively featureless continua of the 0 and $B$ stars acts to effectively hide the presence of lines from the older stars of the host galaxy. Consequently, spectroscopy yields very little information regarding the stellar content of the BCD host galaxy. On the other hand, the nebular spectra do allow for the accurate determination of the abundances in the ionized gas.

- Gas rich. The typical BCD contains a large amount of $H I$ gas. The mean value of $\mathrm{M}_{H I} / \mathrm{M}_{\text {tot }}$ for a sample of $122 \mathrm{BCDs}$ is 0.16 (Salzer et al. 1999a). Figure 1 shows the distribution of $M_{H I} / L_{B}$ for this sample, plotted vs. absolute magnitude. The open symbols represent average values for spiral and ir regular galaxies taken from the literature. A statistical correction to the luminosities and mass-to-light ratios of the BCDs has been applied to account for the fact that their luminosities are elevated by an average of $0.75 \mathrm{~B}$ magnitudes due to the starburst (see below). Thus, the figure shows where the host galaxies of the BCDs would lie in this diagram if the starburst component were removed. On average, the BCD hosts have a factor of $\sim 2$ higher $\mathrm{HI}$ gas mass at a given absolute magnitude than do the more quiescent irregular galaxies with which they are compared. To be sure, there are some BCDs with low HI gas content, but on the whole, BCDs are quite gas rich.

The presence of many BCD hosts lying above the trend set by the comparison sample could be interpreted in two ways: either they have unusually high HI masses, or they have normal HI masses but unusually low luminosities. The presence of a population of galaxies in the upper portion of this diagram, with no counterpart in the comparison sample, suggests the possibility that these galaxies would be difficult to detect in typical galaxy surveys during their quiescent 


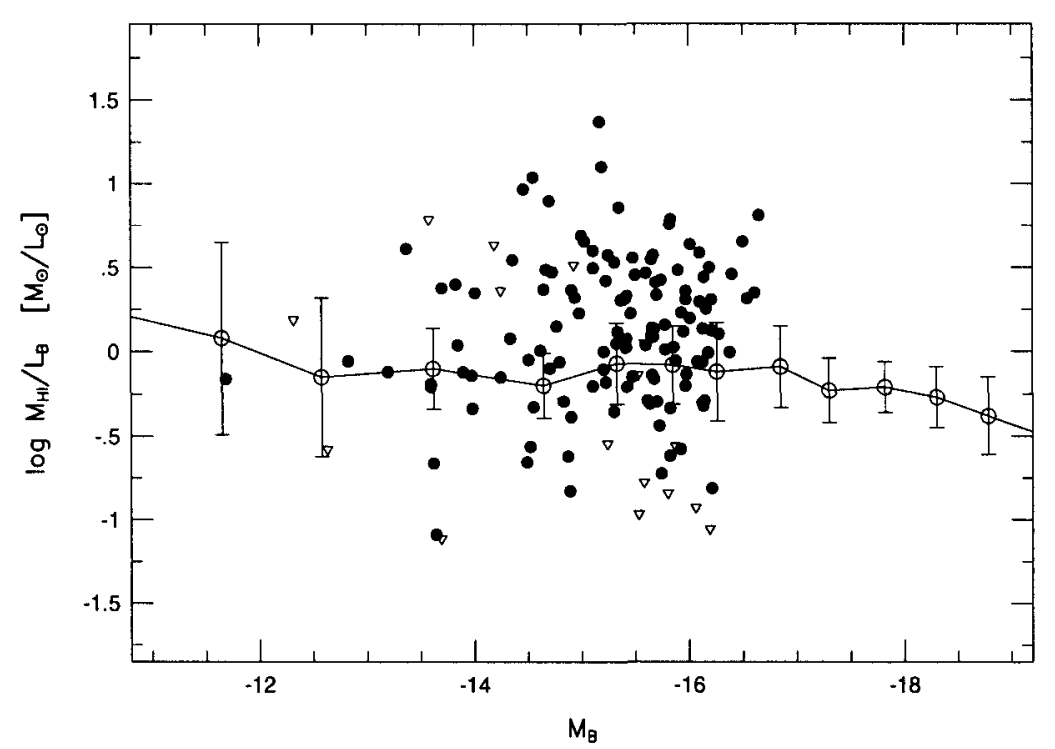

Figure 1. The HI content of BCDs. Here we plot the HI mass to blue-light ratio vs. absolute magnitude for a large sample of BCDs taken from Salzer et al. 1999a. The BCDs are shown as filled circles, while open triangles show upper limits for the BCDs not detected in $\mathrm{HI}$. The connected open circles are the mean values of $\mathrm{M}_{H I} / \mathrm{L}_{B}$ for a sample of spiral and irregular galaxies taken from the literature. After correcting the BCDs for the excess light due to their starburst, they are seen to mostly lie at or above the location of the normal galaxies in this diagram.

phases. This led us to suspect originally that the BCD hosts might in fact be LSB dwarfs.

- Most not bursting for the first time. The dominance of the starburst led a number of authors to suggest that the observed burst of star formation in some BCDs represents the first episode of star formation in these objects (e.g., Searle \& Sargent 1972). However, more recent imaging studies have shown fairly convincingly that nearly all BCDs do possess an older, underlying population of stars (e.g., Papaderos et al. 1996a,b; Telles \& Terlevich 1997). There are a few galaxies, such as I Zw 18 and SBS 0335-04, for which a strong case can be made that the current starburst represents the first major episode of star formation (e.g., Thuan et al. 1997), but these are the exception rather than the rule. Although it is virtually impossible to assign ages to the underlying 
population, one can say with some confidence that most BCDs began forming stars long ago.

- Burst strengths not extreme. Because of the optical dominance of the starburst component in BCDs, it is often assumed that the current burst involves a large fraction of the mass of the galaxy, and that it has elevated the brightness of the galaxy by a large amount (estimated at 3-5 magnitudes by some authors). However, this turns out to be an overestimate. Modeling of the starbursts in over a dozen extreme BCDs shows that, on average, only a few percent of the available $\mathrm{HI}$ gas is being used in the current starburst (Salzer et al. 1999b). Further, this work shows that the average B-band luminosity enhancement due to the starburst (both stellar and nebular contributions) is only 0.75 magnitudes. Thus, the "bursts" in BCDs are not such extreme events as one might think.

\section{Some Recent Key Results}

Three recent results have played a significant role in reshaping our view of the nature of $\mathrm{BCD}$ host galaxies (or at least in how we might interpret the available data).

The first of these are theoretical studies which attempt to account for the fate of the ISM in dwarf galaxies which experience a major starburst. Early work suggested that even modest numbers of supernovae were enough to completely remove the ambient gas in small galaxies. One implication of this was that BCDs would lose all of their gas following the starburst event, and after 1$2 \mathrm{Gyr}$ resemble dwarf ellipticals. However, more recent studies (DeYoung \& Heckman 1994; MacLow \& Ferrarra 1998; Brighenti \& D'Ercole 1999) have come to the opposite conclusion: only for extremely low-mass galaxies does a starburst remove all of the gas. These new simulations suggest that the hot SN ejecta (including most of the metals produced in the high mass stars) will escape, while the bulk of the colder ISM will remain. If correct, these new studies change drastically our view of post-BCDs. This result is also consistent with the picture that BCDs have had previous star formation. If dwarf galaxies lost their gas easily due to SN outflows, then they would have trouble creating additional generations of stars. Since most BCDs are known to possess at least two generations of stars (and perhaps many more), the ability to retain their gas is obviously crucial.

Another recent finding that may play a major role in our understanding of the $\mathrm{BCD}$ phenomenon is that BCDs not only have more HI gas than comparablesized dIs, but the gas is more centrally concentrated. In a recent paper by van Zee et al. (1998), the azimuthally-averaged HI distributions for 8 BCDs were compared to those for a similar number of dIs. The BCDs tend to have strongly peaked gas distributions, i.e., a large reservoir of $\mathrm{HI}$ in the central portions of the galaxies. We believe that this is related to the presence of the starbursts in the BCDs, and in fact may be a necessary condition for the occurrence of a strong, sustained star-formation episode like that seen in BCDs.

The third result which has had a major impact on our view of BCD hosts, and which as the primary motivation for carrying out the current study, is the 
recent work by Papaderos et al. (1996a,b) and Telles \& Terlevich (1997). These studies investigate the surface brightness distributions of BCDs, and shed new light on the nature of the host galaxies of BCDs. In particular, Papaderos et al. utilized surface photometry of BCDs out to faint surface brightness levels which allowed them to study both the distribution of the starburst light as well as the light from the underlying host galaxy. Among their results was the suggestion that the host galaxies of BCDs have significantly different characteristics than other, more normal, dwarf galaxies. Their success gave us the incentive to carry out a similar analysis on a large number of existing BCD images obtained previously for other purposes (Salzer \& Elston 1992; Salzer et al. 1999b).

\section{Structural Parameters of BCD Host Galaxies}

We have carried out detailed surface photometry using deep B-band CCD images for a sample of $18 \mathrm{BCDs}$ and $11 \mathrm{dIs}$. The BCDs were selected from a variety of survey lists, and were chosen to represent the subsample of dwarf star-forming galaxies with the most extreme properties (i.e., the most intense star-formation events). The dI galaxies were analyzed as a comparison sample. These galaxies are part of a separate study of the properties of nearby dwarf galaxies known to exhibit numerous holes in their HI distributions (see Rhode et al. 1999, this volume). Additional comparison dIs were taken from the study by Patterson \& Thuan (1996). Both the BCD and dI samples were limited to galaxies with $\mathrm{M}_{B}$ $>-17$.

Since the BCDs are dominated by a (usually) central starburst, isophotal fitting is a tricky business. In general, it is not trivial to distinguish between light from the underlying host galaxy and light from the starburst. However, since we were not particularly interested in the starburst region, we adopted the following simple approach. First, we allowed our isophote-fitting software to fit the entire galaxy with no preset restrictions (i.e., we did not exclude the starburst region). We then used the radial brightness profiles derived from the isophote fitting to determine the structural parameters of the galaxy: central surface brightness $\left(\mu_{0}\right)$ and exponential scale length $(\alpha)$. We assumed that the underlying host galaxy could be represented by a single exponential profile. The exponential disk profile was fit only in the outer portions of the galaxy, well outside the radius occupied by the starburst. We used $\mathrm{H} \alpha$ images of each galaxy to define where the star formation was occurring. In this way, we can be fairly confident that the derived parameters represent those of the underlying galaxy, and are not affected by the intense starburst. For consistency, we carried olit fits to the radial brightness profiles of the comparison dIs in the exact same way. Only the outer portions of the dI profiles were used to determine the structural fits. Complete details of our analysis are given in Norton \& Salzer (1999).

The results of our structural parameter determinations are shown in Figures 2 and 3 . Figure 2 plots the extrapolated central surface brightness vs. absolute magnitude, while Figure 3 shows disk scale length plotted against absolute magnitude. Also plotted are similar quantities for BCDs from Papaderos et al., and additional dls from Patterson \& Thuan. The host galaxies of BCDs lie at the extremes of the dwarf irregulars in both plots, in the sense that the BCDs have systematically higher central surface brightnesses and smaller disk scale 


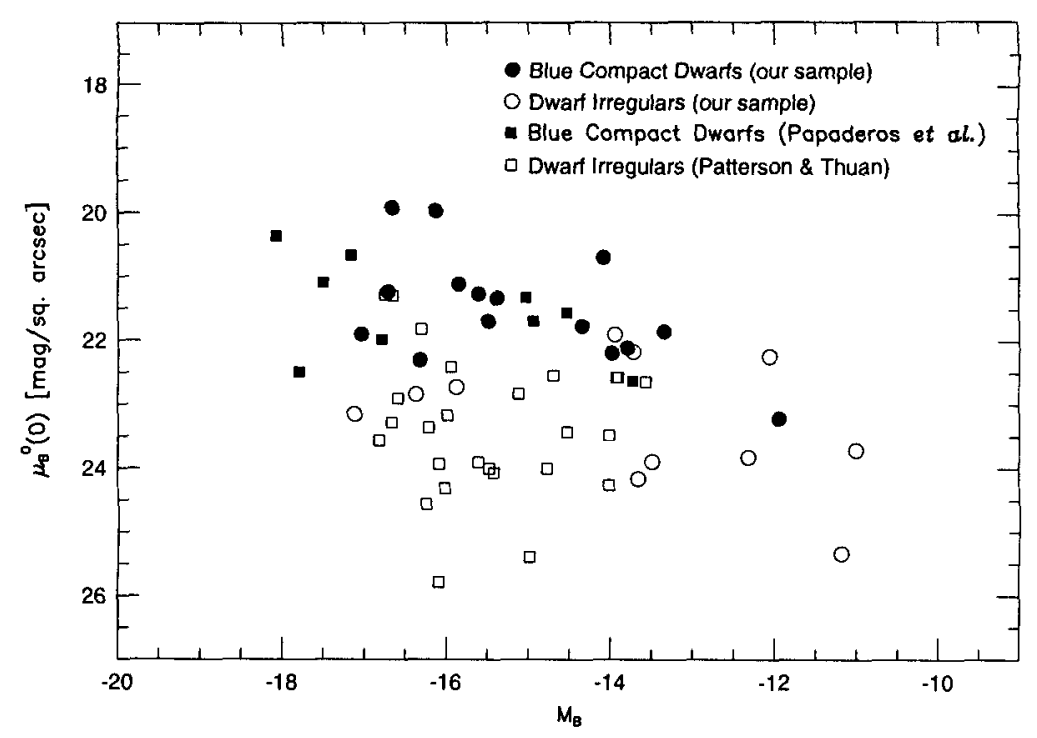

Figure 2. Plot of central surface brightness vs. absolute magnitude for our sample of BCDs and dIs. Also plotted are BCDs from Papaderos et al. and dIs from Patterson \& Thuan. The BCDs have systematically higher central surface brightnesses than do the dIs.

lengths. That is, the hosts of BCDs are significantly more compact, and possess higher central mass densities than do more quiescent dIs. We stress that our measurements for the BCDs are done in a way that ignores the light from the starburst - the quantities plotted are for the underlying host galaxies only. If the light from the BCD were included, the difference between the BCDs and $\mathrm{dIs}$ would be even greater.

\section{Discussion: Implications for Dwarf Galaxy Evolution}

The results of our surface photometry, when combined with the recent studies mentioned in Section 3, can now be used to address the question posed in the introduction: are BCDs just typical low-surface-brightness dI galaxies currently in a bursting state? We believe that the answer to that question is a definite NO!

The typical BCD appears to be hosted by a gas-rich galaxy with structrual parameters at the extreme end of those exhibited by dwarfs. On average, BCDs are hosted by centrally concentrated systems, with very small scale lengths and high central mass densities. If there is a continuum of values for the scale length 


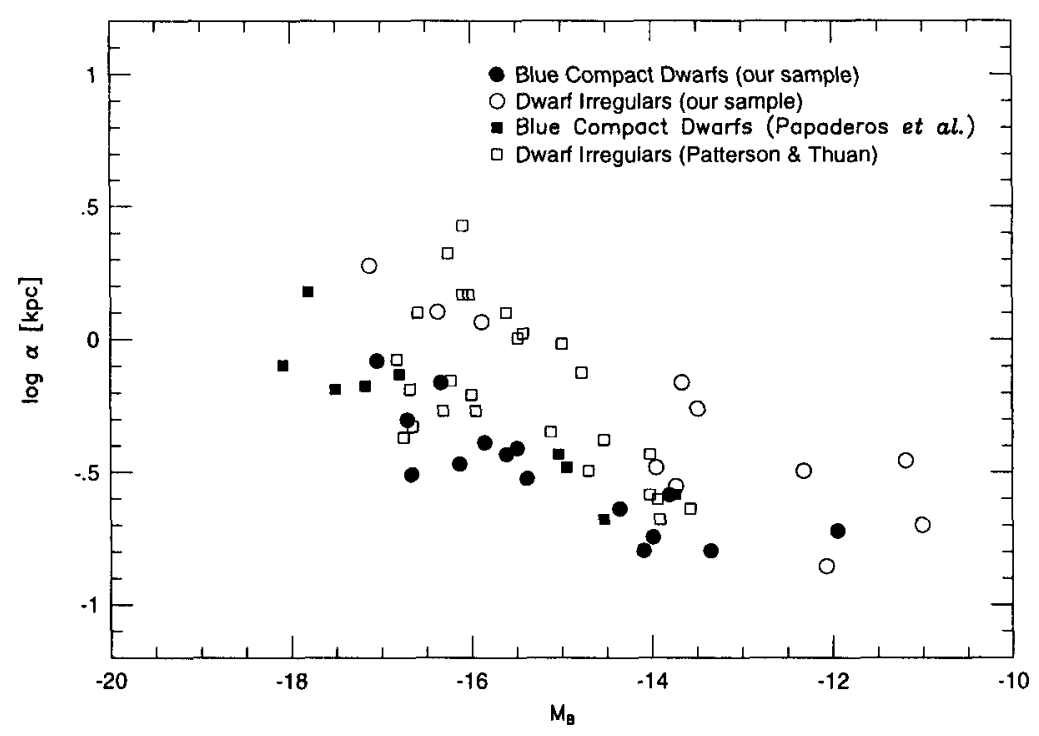

Figure 3. Plot of the exponential disk scale length $(\alpha)$ vs. absolute magnitude for our sample of BCDs and dIs. Also plotted are BCDs from Papaderos et al. and dIs from Patterson \& Thuan. The BCDs tend to possess smaller disk scale lengths than the dIs, indicating a more compact distribution for the underlying stars.

and central surface brightness for dwarf galaxies, BCD hosts occupy the extreme end of the distribution. If this picture is correct, then BCDs cannot be hosted by just any dwarf galaxy. Rather, they are preferentially found in the most centrally concentrated systems. Further, they appear to occur in systems with the highest central HI gas densities. This makes perfect sense, since it would be exactly these systems which favor repeated star-formation bursts, and which could build up enough gas mass in a restricted area to generate a star-formation episode of sufficient magnitude to be called a BCD.

This scenario has a number of important implications for our views on how dwarf galaxies evolve. If $\mathrm{BCDs}$ and other dwarfs do not lose most of their gas mass as a result of a starburst, as suggested by the simulations of MacLow \& Ferrara (1998), they will not evolve into gas-poor $\mathrm{dE}$ galaxies, as some authors have contended. Further, since little mass is ejected, there will be no significant dynamical relaxation of the system. In other words, the structural parameters will not change significantly after the starburst is over. Rather, the post-burst $\mathrm{BCDs}$ will remain compact. By remaining in such a configuration, with high central mass densities and high HI gas content, the period of time between bursts for a $\mathrm{BCD}$ could be quite short, probably on the order of the time scale 
for the cold ambient gas to settle down after the effects of the last burst are over (perhaps on a time scale of $\sim 100 \mathrm{Myr}$ ). If correct, this implies a very high duty cycle for BCDs. It also helps to explain why we don't see large numbers of compact, post-burst dwarf galaxies. First of all, most dwarfs cannot become BCDs in the first place, and those that do might spend a large fraction of their time in a bursting phase.

However one decides to interpret the current results, it seems clear that the old picture of dwarf galaxy evolution, where most or all gas-rich dwarfs go through one or more BCD-like episodes, is not correct. Rather, only a special subsample of the dwarf galaxy population can host a starburst of the magnitude that would qualify it as a BCD. This simple result requires substantial rethinking of our view of how dwarfs galaxies evolve, since in this scenario most galaxies do not evolve through the $\mathrm{BCD}$ phase. Thus transitions from gas-rich dIs to gas-poor dEs seem much less likely, and the galaxies which are currently seen as BCDs will not evolve into either normal dIs or gas-poor dEs after the starburst phase is over. A corollary to this is that galaxies currently seen as low-surfacebrightness dwarfs have most likely never gone through a BCD-like phase, and probably never will.

Acknowledgments. We are grateful to our many collaborators on our various dwarf galaxy projects for input and suggestions. In particular, thanks to Liese van Zee, Katherine Rhode, David Westpfahl, and David Sudarsky. JJS gratefully acknowledges financial support from the National Science Foundation.

\section{References}

Brighenti, F., \& D'Ercole, A. 1999, in preparation

De Young, D.S., \& Heckman, T.M. 1994, ApJ, 431, 598

MacLow, M.-M., \& Ferrara, A. 1998, ApJ, in press

Norton, S.A., \& Salzer, J.J. 1999, in preparation

Papaderos, P., Loose, H.-H., Fricke, K.J., \& Thuan, T.X. 1996a, A\&A, 314, 59

Papaderos, P., Loose, H.-H., Thuan, T.X., \& Fricke, K.J. 1996a, A\&AS, 120, 207

Patterson, R.J., \& Thuan, T.X. 1996, ApJS, 107, 103

Rhode, K.L., Salzer, J.J., \& Westpfahl, D.J. 1999, this volume

Salzer, J. J., \& Elston, R. 1992, in I.A.U. Symposium No. 149, ed. B. Barbuy and A. Renzini (Dordrecht: Kluwer), p. 482

Salzer, J.J., Rosenberg, J.L., Weisstein, E.W., Mazzarella, J.M., \& Bothun, G.D. 1999a, in preparation

Salzer, J. J., Sudarsky, D.L., \& Elston, R. 1999b, in preparation

Searle, L. \& Sargent, W.L.W. 1972, ApJ, 173, 25

Telles, E., \& Terlevich, R. 1997, MNRAS, 286, 183

Thuan, T.X., Izotov, Y.I., \& Lipovetsky, V.A. 1997, ApJ, 477, 661

Thuan, T.X., \& Martin, G.E. 1983, ApJ, 247, 823

van Zee, L., Skillman, E.D., \& Salzer, J.J. 1998, AJ, 116, 1186 